\title{
Wireless Sensor Network for Monitoring Physical Variables Applied to Green Technology (IoT Green Technology)
}

\author{
Gregorio Trinidad Garcia, Victoria Meis Sanchez, Carmen Nora Lopez Marin, \\ Jose Italo Cortez, Carlos A. Rios Acevedo, Griselda Saldaña Gonzalez, \\ Jose L. Hernandez Ameca, and Maria del C. Molina Garcia
}

\begin{abstract}
In this article an experimental system of sensor network for monitoring physical variables applied to green technology in conjunction with internet of things (IoT) is presented. This, in a controlled ambient, that can guarantee the development and growth of the plants (exemplary/specimen). This application is focused on agriculture. Using Wireless Sensor Network (WSN) has a promising future due to current technical advances and its almost unlimited applications. This work propose WSN topologies, the measurement methodology, the sensors distribution and the visualization of the registered data in function of the area monitored, the communication protocol established by $\mathrm{Wi}-\mathrm{Fi}$ and the reads of the environmental temperature (ET), environmental humidity (EH) and ground humidity (GH) are registered and displayed in the web, during the registry of this measurements the data is analyzed and processed to perform relevant actions oriented to the control of physical variables at the monitored environmental area. Monitoring of the physical environmental conditions is registered every hour in a period of 24 hours. It is proposed to apply the system to green technologies together with internet of the things.
\end{abstract}

Index Terms - Data Transfer; Internet of Things; IoT Green Technology; Real-Time System; Sensor System; Wireless Sensor Networks.

\section{INTRODUCTION}

The Integration of sensor networks in wireless

Published on February 13, 2018.

Gregorio Trinidad García is with the Computer Science Faculty at Benemerita Universidad Autonoma de Puebla, C.U., 72000 Puebla, México (e-mail: tgarcia@cs.buap.mx).

Victoria Meis Sanchez is with the Computer Science Faculty at Benemerita Universidad Autonoma de Puebla, C.U., 72000 Puebla, México (e-mail: victoria.meis@ alumno.buap.mx).

Carmen Nora Lopez Marin is with the Computer Science Faculty at Benemerita Universidad Autonoma de Puebla, C.U., 72000 Puebla, México (e-mail: cnora.lomarin@itesm.mx).

Jose Italo Cortez is with the Computer Science Faculty at Benemerita Universidad Autonoma de Puebla, C.U., 72000 Puebla, México (e-mail: italo.cortez@lisder.cs.buap.mx).

Carlos A. Rios Acevedo is with the Computer Science Faculty at Benemerita Universidad Autonoma de Puebla, C.U., 72000 Puebla, México (e-mail: carlos.riosa@correo.buap.mx).

Griselda Saldaña González is with the Mecatronics Division at Universidad Tecnologica de Puebla, Antiguo Camino a la resurrección, 72300 Puebla México (e-mail: griselda.saldana@utpuebla.edu.mx).

Jose L. Hernandez Ameca is with the Computer Science Faculty at Benemerita Universidad Autonoma de Puebla, C.U., 72000 Puebla, México (e-mail: joseluis.hdzameca@correo.buap.mx).

Maria del C. Molina Garcia is with the Computer Science Faculty at Benemerita Universidad Autonoma de Puebla, C.U., 72000 Puebla, México (e-mail: consuelo.molina@correo.buap.mx). communication system has generated an area of interest in applications such as industrial plants and the agricultural sector among others. In general, in those systems where a constant momitoring of physical signal is required, a sensor network whit wireless technology in applicable.

The wireless systems present several advantages in comparison to wired technologies such as low cost, lower energy consumption, mobility and portability.

The use of integrated sensors into wireless networks has a promising future, as it is the case of the Internet of Things (IoT), this allows the global interconnection of heterogeneous physical objects with advanced functionality. This emerging technology is evolving towards intelligent environments, where the technologies of wireless networks and access to information play a key role in process control, signal monitoring, environment control, along with others.

The technological advance in the last years has allowed integrating technology in the daily activities of the human being, one of these the care of the environment [1].

As main activity, it is the generation of controlled environments to promote the growth of plants, using the monitoring and control of the environmental humidity, ground humidity and environmental temperature due to these variables are vitally important in agriculture.

The use of sensors to monitor physical variables of interest and the use of actuators allow the interaction with the environment, giving control of the growth area and development of the plants [2]. Applying sensors and using suitable techniques for recording of signals, it is possible to monitor large areas of land.

Monitor in real-time the physical variables and apply the so-called internet of things, presents an interesting technological challenge. In the near future an exponential growth and a direct impact in the daily live is expected [3].

In this work a system that monitors the environmental humidity, environmental temperature and ground humidity by means of modules is presented. These modules are distributed in strategically points to take measurements, which is an innovative application in the green technology joined to the Internet of the things (IoT Green) [4].

Development modules or cells conformed for an area that delimits the specimen (plant) of particular interest, with a set of sensors and Wi-Fi connection with the $802.11 \mathrm{~b} / \mathrm{g} / \mathrm{n}$ Wireless protocol [5] are proposed.

Modularity provides to the system the capability to be adapted to the needs required by the application in particular. 


\section{PRoblem Statement}

A controlled environmental, promotes the adequate growth of plants. As a first phase of the system, the physical variables under monitoring are the ground humidity, environmental humidity and temperature, these parameters directly affect growth and development of the plant. Each module provides the average information of the samples taken from environmental physical signals for a set of specimens (plants) distributed in a larger area.

The reading of the physical environmental parameters from sensors, their registry and visualization and their interpretation has been solved with a new-fangled approach to new technologies, in this case making use of the internet of the things set to green technologies [6].

The proposed design consists of modules placed in specific areas, each module contains a ground humidity (GH) sensor, an environmental humidity (EH) sensor and an environmental temperature (ET) sensor [7]. The periodicity for recording these signals is a function of the type of specimen (plant) of interest and the environmental conditions in which it grows.

The Wireless Sensor Network (WSN) proposed, monitors the physical signals of the environment in each module, at intervals of one hour, which guarantees continuous recording during a period of complete insolation.

The information recorded for the sensors is sent via $\mathrm{Wi}-\mathrm{Fi}$ through an access point [8]. Later it can be displayed at any access point using a password.

According to the registered data, they are modified and controlled by applying the appropriate ground humidity or modifying the humidity and temperature of the environment.

\section{EXPERIMENTAL ARRANGEMENT}

The proposed experimental arrangement for monitoring the environmental physical variables consists of modules; each module is composed of any specimen (plant), ground humidity sensor, environmental humidity sensor and environmental temperature sensor. Fig. 1 shows a diagram of the arrangement and organization of an individual module.

The ground humidity sensor is placed at $20 \mathrm{~cm}$ below the surface; this is due to the root of the plant. For the recording of the ambient temperature, the sensor was fixed at $30 \mathrm{~cm}$ from the floor surface; the humidity sensor is placed at the same height [9].

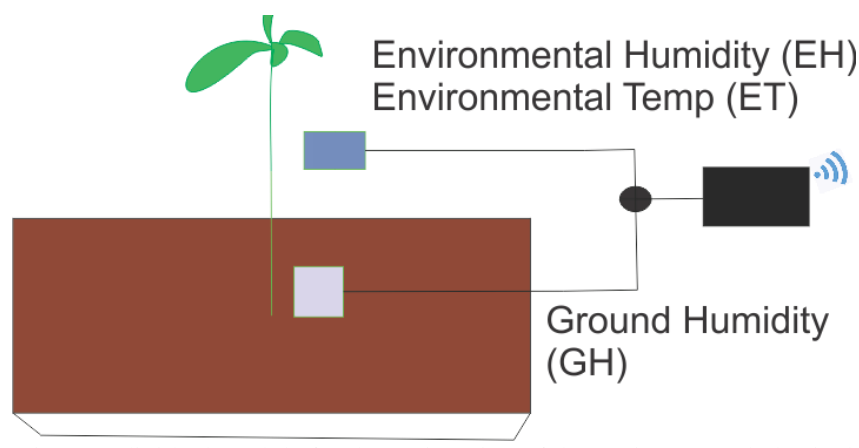

Fig. 1. Individual module for the registration of the environmental physical variables for a specimen (plant).

The number of modules depends on the type of specimens to be monitored and the area under observation. Fig. 2 illustrates the proposal for the distribution of a set of modules in an area $\mathrm{A}_{1}$.

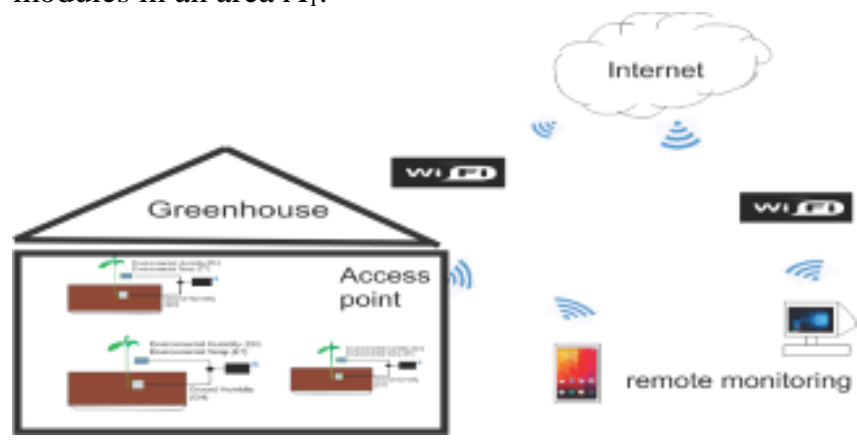

Fig. 2. Distribution of the modules for a Surface A1, (Network architecture star topology).

The measuring of the environmental physical variables performed by the sensors in each module is obtained in the same time span [10]. When verifying the reading recorded by the set of sensors in each module, a string with all the concatenated values is generated [11]. Using a wireless module, the data is sent to a web page where they are processed using PHP through the GET method.

The System works autonomously and can be used to monitor inaccessible areas, to strengthen surveillance and environmental research.

\section{A. Ground humidity transducer}

The ground humidity transducer is a component of two conductive plates separated $d$ centimeters. If there is moisture in the soil conductivity is generated between the plates, the conductivity increases as a function of the percentage of moisture between conductive flat plates.

The change in the percentage of soil moisture generates a variation of conductivity. This increment or decrement in conductivity generates the variation of direct electric current between parallel flat plates.

Fig. 3 shows the transducer used in the experimental arrangement.

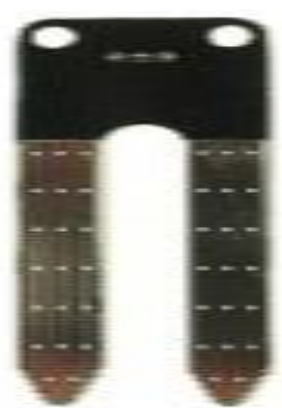

Fig. 3. Conductive flat plate transducer

The variation in conductivity is proportional to the percentage of moisture contained in the soil. A percentage of $100 \%$ is considered the total humidity.

The soil moisture meter shows the current humidity in absolute percentage, as well as the degree of saturation of the soil. It must be taken into account that in addition to the solid components, air is also found in the ground; the percentage varies according to the soil type. Thick-textured soils contain less air (approximately 40\% vol.) than fine- 
textured soils (up to $60 \%$ vol.). If the percentage of air is higher in the soil, then the density of the soil is lower and the air can be substituted by water. When the water substitutes all the air in the soil, there is a saturated soil. Under normal conditions the ground is not able to retain all the water, since it is filtered.

\section{B. Temperature sensor and environmental moisture}

One of the physical variables that should be known and measured is the relative humidity of the air. This physical variable is of main importance in sectors such as industry, transport and horticulture, among others. Similarly, the temperature of the environment plays a vital role in such sectors. These two variables are key to the application of the wireless sensor network proposed in this work.

A wide variety of sensors and systems are available for the measurement of temperature and humidity. In this application, a common sensor is used.

Given the characteristics of the proposed system and as a prototype, the sensor used to record the temperature and humidity is DHT11. The sensor is inexpensive, has modular features and it is easy to implant. The requirements of the prototype are met with this sensor.

\section{System implementation}

Fig. 4 shows an implemented module of the Wireless Sensor Network (WSN) set.

The module consists of the specimen (plant), the ground humidity sensor, environmental temperature sensor and environmental humidity sensor [12]. The sensors are connected to the control system where the signals are conditioned and sent to the web through an access point.

The wireless sensor network uses the communication protocol based on the IEEE 802.11 standard to communicate the sensors nodes via Wi-Fi. It uses $2.4 \mathrm{GHz}$ in the radiofrequency spectrum. This technology has advantages such as transmission speed that can reach $11 \mathrm{Mbps}$; an automatic adjustment in bandwidth; low interference, it can be adjusted to $1 \mathrm{Mbps}, 2 \mathrm{Mbps}$ or $5 \mathrm{Mbps}$; a considerable coverage area; great distance of transmission effectiveness; avoids wiring, flexible network structure and good mobility.

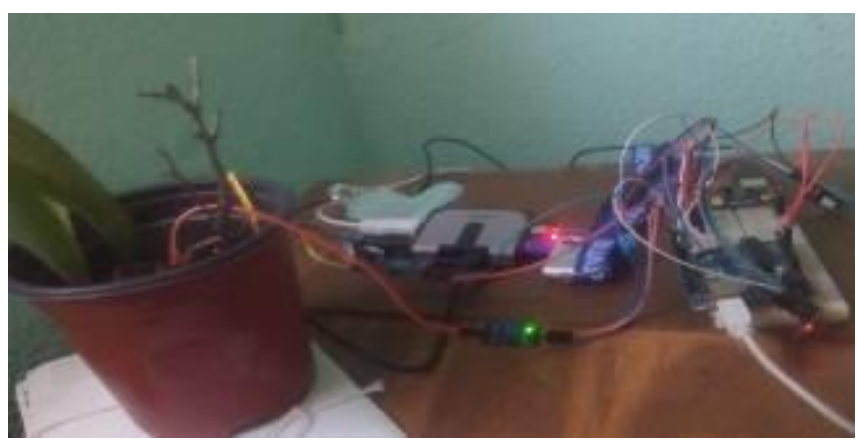

Fig. 4. Image of experimental arrangement.

The registered data are sent using PHP and jquery commands through the GET method to a web page where they are stored [13].

\section{EXPERIMENTAL RESULT}

In the proposed WSN topology, each module consists of a set of sensors and each sensor registers an environmental physical variable [14]. The designed control system multiplexes the reading control signal to a single module at the time to read the data recorded by each sensor. The control generates a reading signal at intervals of one-hour to collect the measurements recorded in each module [15]. Measurements are directed through a wireless device to an access point.

The data registered in each module are displayed on a web page at a remote point and are accessed through a password. The behavior of the remaining modules is similar.

\section{A. Comparison and system calibration}

As a validation for the measurements recorder by the DHT11 sensor, a comparative table of the environment temperature measurements recorder by three different sensor DHT11, DS18B20 and LM355 is shown in Table I. The following table shows a sample of readings recorded over the same period of time.

TABLE I: TEMPERATURE RECORDING

\begin{tabular}{|c|c|c|c|}
\hline $\begin{array}{l}\text { Time } \\
\text { Hours }\end{array}$ & $\begin{array}{c}\text { DHT11 } \\
{ }^{\circ} \mathrm{C}\end{array}$ & $\begin{array}{c}\text { DS18B20 } \\
{ }^{\circ} \mathrm{C}\end{array}$ & $\underset{{ }^{\circ} \mathrm{C}}{\mathbf{L M 3 3 5}}$ \\
\hline $11: 30$ & 22.00 & 22.71 & 23.15 \\
\hline $12: 25$ & 22.00 & 22.72 & 22.90 \\
\hline $13: 15$ & 22.00 & 22.70 & 23.10 \\
\hline $14: 20$ & 23.00 & 23.80 & 24.00 \\
\hline $15: 15$ & 23.00 & 23.20 & 23.80 \\
\hline $16: 00$ & 22.00 & 22.50 & 22.78 \\
\hline $16: 45$ & 23.00 & 23.60 & 23.95 \\
\hline $17: 50$ & 23.00 & 23.60 & 23.80 \\
\hline $18: 30$ & 22.00 & 22.90 & 23.00 \\
\hline $19: 45$ & 21.00 & 20.80 & 21.20 \\
\hline
\end{tabular}

For the application being reported, the data recorded are within tolerant limits [16], [17]. This validates the measurements recorded by the proposed Wireless Sensor Network (WSN). The temperature reported by Weatheronline is shown in Fig. 5.

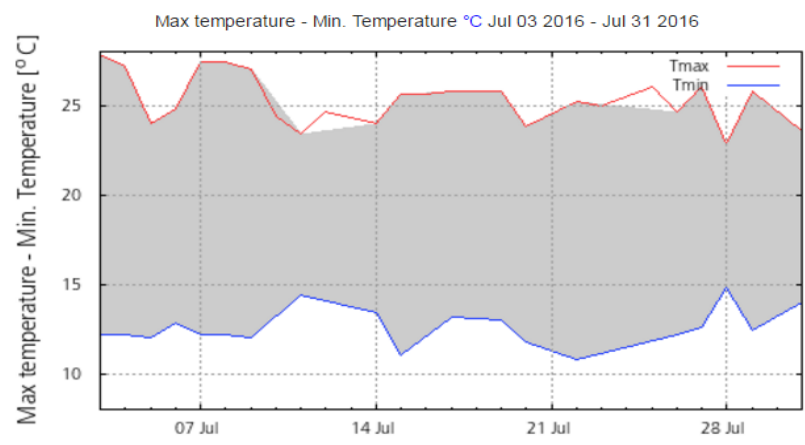

Fig. 5. Graphic with report of rank of temperature reported by weatheronline.

The graphic in Fig. 5 shows the behavior of the temperature in a period of three weeks. In particular, the maximum temperature recorded on July 13, 2016 is within the range registered by the wireless sensor network (WSN) reported in this paper.

\section{B. Algorithm for the proposed model}

For the reported application, it is not required a continuous monitoring of environmental physical signals, 
samples with a frequency of one hour and a period of 24 hours are enough [18]. The proposed algorithm for this Wireless Sensor Network (WSN) system is:

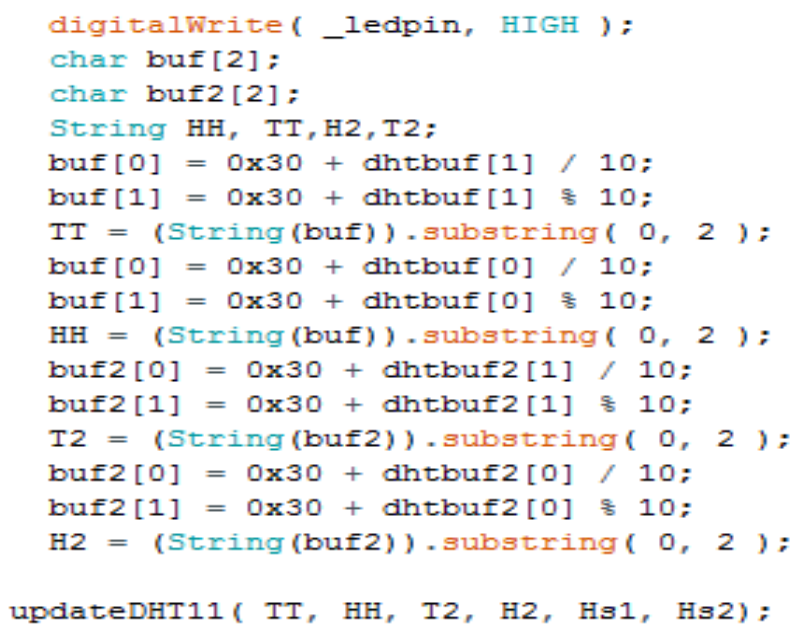

\section{Relative humidity}

Relative humidity $\mathrm{RH}[\%]$, is the proportion of actual water vapor in the air compared to the amount of water vapor required for saturation at the corresponding temperature. It indicates how close the air is to saturation. Fig. 7 shows the image of the relative humidity chart by weatheronline. It is measured in percentage between 0 and 100 , where $0 \%$ means completely dry air and $100 \%$ saturated air.

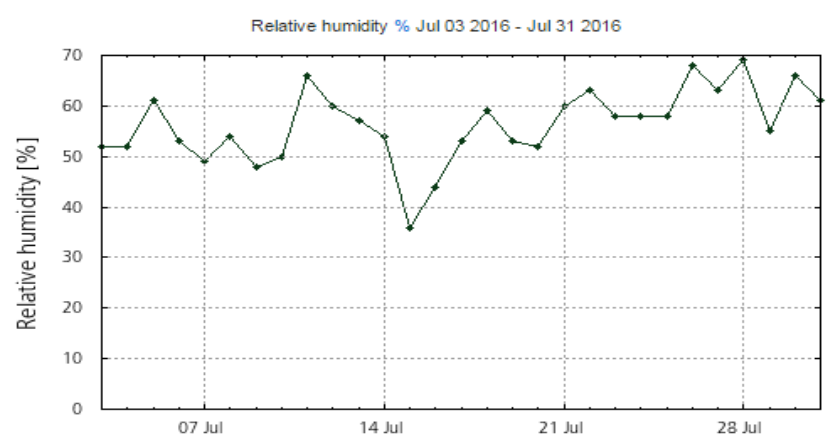

Fig. 7. Image of the graphic report by Weatheronline of relative humidity

\section{Registered date for the wireless sensor network (WSN) proposed}

Table II lists the data of the measurements recorded by the first module (MO1). Physical variables are monitored over a 24-hour period, taking data at one-hour intervals [19]. The physical variables monitored are: environmental temperature (Temp), environmental humidity (HR) and ground humidity.

TABLE II: REGISTERED DATA (MOD1)

\begin{tabular}{|c|c|c|c|}
\hline $\begin{array}{l}\text { Time } \\
\text { Hours }\end{array}$ & $\begin{array}{l}\text { Temp } \\
\text { MO1 }\end{array}$ & $\begin{array}{c}\text { HR [\%] } \\
\text { MO1 }\end{array}$ & $\begin{array}{c}\text { Ground } \\
\text { Humidity [\%] } \\
\text { MO1 }\end{array}$ \\
\hline 11:34:11 & 22 & 46 & 53.91 \\
\hline $12: 28: 23$ & 22 & 48 & 52.44 \\
\hline $13: 22: 30$ & 22 & 48 & 42.09 \\
\hline $14: 16: 36$ & 23 & 46 & 42.68 \\
\hline
\end{tabular}

\begin{tabular}{llll} 
15:10:43 & 23 & 45 & 43.36 \\
$16: 04: 51$ & 25 & 44 & 43.55 \\
$16: 58: 58$ & 23 & 47 & 43.75 \\
$17: 53: 05$ & 23 & 47 & 44.14 \\
$18: 47: 11$ & 22 & 57 & 44.63 \\
$19: 41: 18$ & 23 & 49 & 44.63 \\
$20: 35: 25$ & 23 & 50 & 44.73 \\
$21: 29: 32$ & 23 & 49 & 44.82 \\
$22: 23: 41$ & 22 & 51 & 44.92 \\
$23: 17: 48$ & 22 & 51 & 43.75 \\
$00: 11: 05$ & 22 & 54 & 45.12 \\
$01: 06: 02$ & 22 & 56 & 45.41 \\
$02: 00: 10$ & 22 & 49 & 45.61 \\
$03: 48: 23$ & 22 & 47 & 45.70 \\
$04: 42:: 31$ & 22 & 47 & 45.80 \\
$05: 36: 38$ & 22 & 47 & 45.90 \\
$06: 30: 44$ & 22 & 47 & 46.00 \\
$07: 24: 51$ & 22 & 47 & 46.00 \\
$08: 18: 58$ & 22 & 47 & 44.82 \\
$10: 07: 14$ & 22 & 47 & 45.21 \\
$11: 01: 21$ & 23 & 46 & 46.09 \\
\hline \hline
\end{tabular}

The table III lists the registered values of the measurements in the second module (MOD2). Similarly, the physical variables monitored are environmental temperature, humidity and ground humidity.

TABLE III: REGISTERED DATA (MOD2)

\begin{tabular}{|c|c|c|c|}
\hline $\begin{array}{l}\text { Time } \\
\text { Hours }\end{array}$ & $\begin{array}{l}\text { Temp } \\
\text { MO2 }\end{array}$ & $\begin{array}{c}\text { HR [\%] } \\
\text { MO2 }\end{array}$ & $\begin{array}{c}\text { Ground } \\
\text { Humidity [\%] } \\
\text { MO2 }\end{array}$ \\
\hline $11: 34: 11$ & 23 & 39 & 48.14 \\
\hline $12: 28: 23$ & 23 & 40 & 43.26 \\
\hline $13: 22: 30$ & 23 & 41 & 42.58 \\
\hline $14: 16: 36$ & 23 & 42 & 42.58 \\
\hline $15: 10: 43$ & 23 & 41 & 42.38 \\
\hline $16: 04: 51$ & 23 & 45 & 42.19 \\
\hline $16: 58: 58$ & 23 & 41 & 42.38 \\
\hline $17: 53: 05$ & 23 & 43 & 42.77 \\
\hline $18: 47: 11$ & 23 & 45 & 42.77 \\
\hline $19: 41: 18$ & 23 & 46 & 42.77 \\
\hline $20: 35: 25$ & 22 & 47 & 42.77 \\
\hline $21: 29: 32$ & 22 & 47 & 43.07 \\
\hline $22: 23: 41$ & 22 & 47 & 43.16 \\
\hline $23: 17: 48$ & 22 & 46 & 42.48 \\
\hline 00:11:05 & 22 & 46 & 43.55 \\
\hline 01:06:02 & 22 & 45 & 43.95 \\
\hline 02:00:10 & 22 & 45 & 44.14 \\
\hline $03: 48: 23$ & 22 & 48 & 44.34 \\
\hline $04: 42:: 31$ & 22 & 44 & 44.43 \\
\hline $05: 36: 38$ & 22 & 44 & 44.53 \\
\hline 06:30:44 & 22 & 45 & 44.63 \\
\hline $07: 24: 51$ & 22 & 44 & 44.73 \\
\hline 08:18:58 & 22 & 44 & 43.55 \\
\hline $10: 07: 14$ & 22 & 47 & 43.92 \\
\hline $11: 01: 21$ & 22 & 48 & 44.82 \\
\hline
\end{tabular}

\section{E. Graphic presentations of recorded measurements}

This section presents graphically the measurements recorded in two modules of the proposed Wireless Sensor Network (WSN) [20]. The rest of the modules behave in a similar way.

The measurements recorded in module one (MOD1) are 
shown in Fig. 8 for the ambient temperature (AT).

\section{DHTI 1}

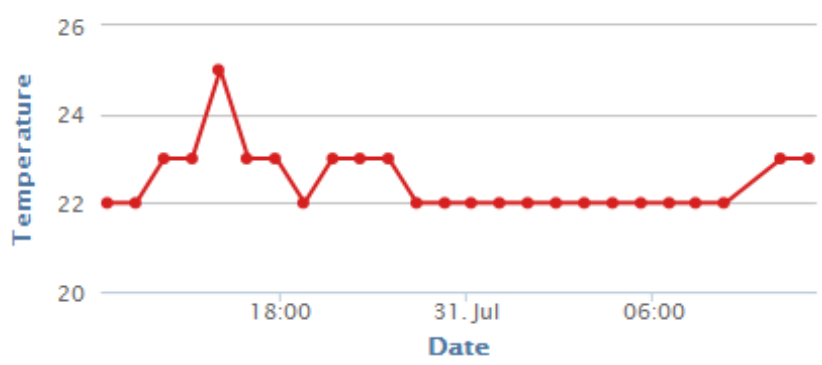

Fig. 8. Ambiental temperature (AT) registration in module one (MOD1)

The environmental humidity recorded by module one (MOD1) is reported in Fig. 9.

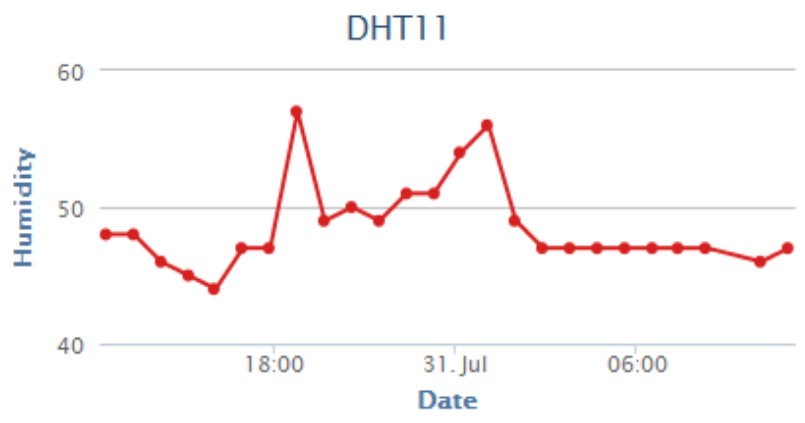

Fig. 9. Environmental humidity record in the module one (MOD1)

The third measurement record corresponds to the ground humidity in module one (MOD1). Fig. 10 shows the graph.

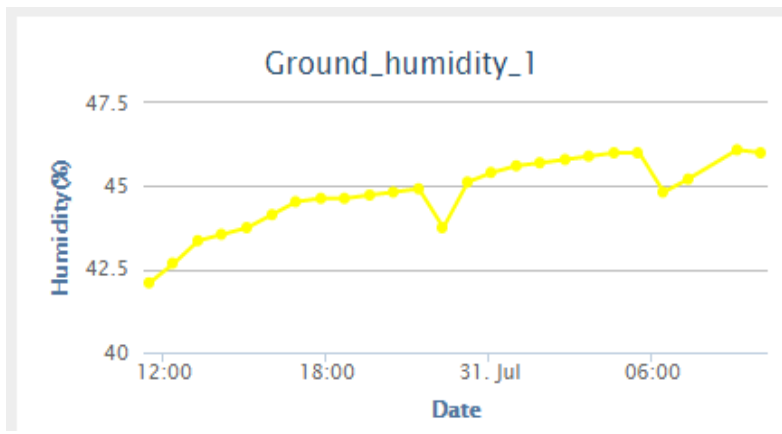

Fig. 10. Ground humidity record in the module one (MOD1)

The remaining modules are similar in relation to the number of sensors and the physical variables monitored. Due to their configuration and topology, they are located at strategic points in the monitored area [21].

The measurements recorded by the sensors of the second module (MOD2) are presented in the following figures.

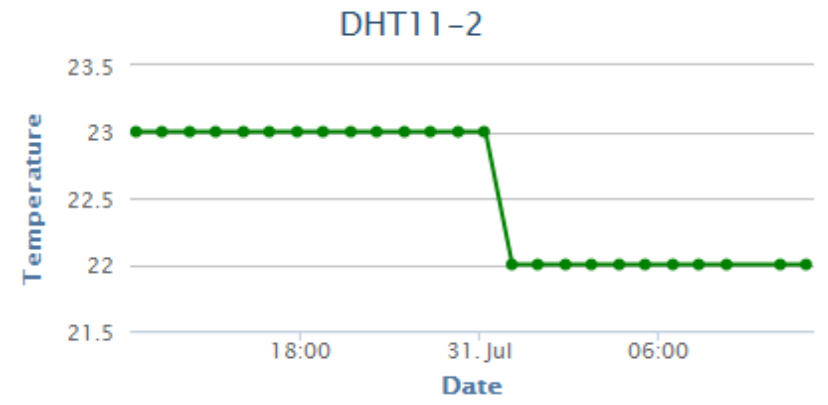

Fig. 11. Environmental temperature record in the module two (MOD2)
DHT11-2

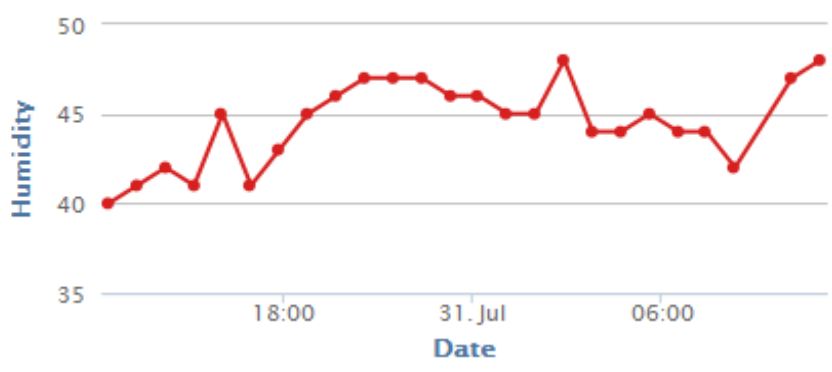

Fig. 12. Environmental humidity record in the module two (MOD2)

Finally, the graphic generated with the data recorded in MOD2 is reported and corresponds to the soil moisture, as shown in Fig. 13.

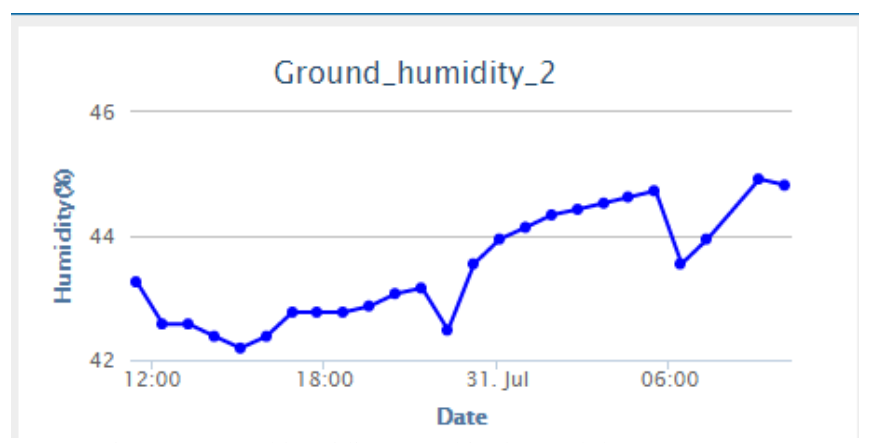

Fig. 13. Ground humidity record in the module two (MOD2)

\section{CONCLUSION}

This article reports the architecture, topology and communication protocol for a network of wireless sensors to monitor environmental physical variables using modules distributed in an area of interest. Due to the proposed topology, the wireless sensor network is scalable and modular. By means of the $802.11 \mathrm{~b} / \mathrm{g} / \mathrm{n}$ communication protocol, an adequate response is obtained for the remote monitoring in real time of the physical environmental variables. The reading recorded by the sensor set in each module is performed in one-hour intervals and in a 24-hour period. As a first result, the information is sent and viewed on a web page for the elaboration and conformation of the functionality to be a classic internet system of things. Due to its application, it gives rise to new experiments in the use of the green technologies and the continuity of the development of new strategies to support the environment. The results obtained in the wireless sensor network propose areas of research in future work related to data encryption, transmission power, processing speed, efficiency, type of modulation, computational cost among others.

The proposed arrangement presents a flexible, economical, modular and easy-to-implement architecture. These features are achieved due to wireless communication and the set of sensors used.

In future work, the number of wireless sensors will be increased to monitor a greater area of interest and provide collaboration tasks between modules, individual data processing and direct transmission to the user, as well as additional information in each module. 


\section{REFERENCES}

[1] Z. Hualin, W. Mengxia, W. Baoyu, H. Jibu and X. Zhiqiang, "Research and development of general data acquisition system based on wireless sensor network dynamic network technology," 2016 IEEE International Conference of Online Analysis and Computing Science (ICOACS), Chongqing.

[2] Y. Li and P. R. Gould, "An experimental wireless sensor system based on low cost, lower power wireless nodes," Wireless Sensor Systems (WSS 2012), IET Conference on, London, 2012, pp. 1-5.

[3] J. P. Dominguez-Morales; A. Rios-Navarro; M. Dominguez-Morales; R. Tapiador-Morales; D. Gutierrez-Galan; D. Cascado-Caballero; A Jimenez-Fernandez; A. Linares-Barranco, "Wireless sensor network for wildlife animals tracking and behavior classifying in Donana," in IEEE Communications Letters , vol.PP, no.99, pp.1-1

[4] M. Stein et al., "Evaluation study for clustering in wireless sensor networks," 2016 10th International Symposium on Communication Systems, Networks and Digital Signal Processing (CSNDSP), Prague, Czech Republic, 2016, pp. 1-6

[5] U. Raza, R. Kharel, M. Hammoudeh, S. Ekpo and B. R. Whiteside, "A service based wireless sensor networks architecture for high valueadded process monitoring," 2016 10th International Symposium on Communication Systems, Networks and Digital Signal Processing (CSNDSP), Prague, Czech Republic, 2016, pp. 1-6

[6] S. Kaur and R. N. Mir, "Base station positioning in Wireless Sensor Networks," 2016 International Conference on Internet of Things and Applications (IOTA), Pune, India, 2016, pp. 116-120.

[7] T. Miyazaki and N. Akiyama, "Formal approach to produce verified programs for wireless sensor nodes," 2016 10th International Symposium on Communication Systems, Networks and Digital Signal Processing (CSNDSP), Prague, Czech Republic, 2016, pp. 1-6.

[8] H. Hadadian and Y. S. Kavian, "Cross-layer protocol using contention mechanism for supporting big data in wireless sensor network," 2016 10th International Symposium on Communication Systems, Networks and Digital Signal Processing (CSNDSP), Prague, Czech Republic, 2016, pp. 1-5.

[9] W. Balid, H. Tafish and H. H. Refai, "Versatile real-time traffic monitoring system using wireless smart sensors networks," 2016 IEEE Wireless Communications and Networking Conference, Doha, Qatar, 2016, pp. 1-6.

[10] S. M. Rakshit, M. Hempel and H. Sharif, "Wireless sensor networks in surface transportation," 2016 10th International Symposium on Communication Systems, Networks and Digital Signal Processing (CSNDSP), Prague, Czech Republic, 2016, pp. 1-6.

[11] N. R. Pandya, V. K. Bhosale and R. M. Autee, "Wireless Sensor Based Handy Patient Monitoring System," 2016 IEEE 6th International Conference on Advanced Computing (IACC), Bhimavaram, 2016, pp. 644-648.

[12] A. Al-Yami, W. Abu-Al-Saud and F. Shahzad, "Simulation of Industrial Wireless Sensor Network (IWSN) protocols," 2016 IEEE Conference on Computer Communications Workshops (INFOCOM WKSHPS), San Francisco, CA, USA, 2016, pp. 527-533.

[13] P. Pandey and V. Laxmi, "Design of low cost and power efficient Wireless vision Sensor for surveillance and monitoring," 2016 International Conference on Computation of Power, Energy Information and Commuincation (ICCPEIC), Chennai, 2016, pp. 113117.

[14] L. Roselli et al., "Review of the present technologies concurrently contributing to the implementation of the Internet of Things (IoT) paradigm: RFID, Green Electronics, WPT and Energy Harvesting," 2015 IEEE Topical Conference on Wireless Sensors and Sensor Networks (WiSNet), San Diego, CA, 2015, pp. 1-3. doi: 10.1109/WISNET.2015.7127402

[15] J. V. V. Sobral, J. J. P. C. Rodrigues, K. Saleem, J. F. de Paz and J. M. Corchado, "A composite routing metric for wireless sensor networks in AAL-IoT," 2016 9th IFIP Wireless and Mobile Networking Conference (WMNC), Colmar, 2016, pp. 168-173. doi: 10.1109/WMNC.2016.7543985

[16] W. Hyun, I. You, J. Jang and F. Y. Leu, "A Wireless Body Sensor Network and Its Applications: Rehearsal with a Smartphone," 2016 10th International Conference on Innovative Mobile and Internet Services in Ubiquitous Computing (IMIS), Fukuoka, Japan, 2016, pp. 415-418.

[17] S. A. Taylor and H. Sharif, "Wearable Patient Monitoring Application (ECG) using Wireless Sensor Networks," 2006 International Conference of the IEEE Engineering in Medicine and Biology Society, New York, NY, 2006, pp. 5977-5980.

[18] N. O'Donoughue, S. Kulkarni and D. Marzella, "Design and Implementation of a Framework for Monitoring Patients in Hospitals Using Wireless Sensors in Ad Hoc Configuration," 2006 International
Conference of the IEEE Engineering in Medicine and Biology Society, New York, NY, 2006, pp. 6449-6452.

[19] D. Parthasarathy, R. Whiton, J. Hagerskans and T. Gustafsson, "An in-vehicle wireless sensor network for heavy vehicles," 2016 IEEE 21st International Conference on Emerging Technologies and Factory Automation (ETFA), Berlin, 2016, pp. 1-8

[20] J. Fourmann et al., "Incident pressure measurement in air blast using wireless sensors," 2016 IEEE International Symposium on Antennas and Propagation (APSURSI), Fajardo, 2016, pp. 1999-2000.

[21] S. Rademacher, K. Schmitt, M. Mengers and J. Wöllenstein, "Sensor network with energy efficient and low-cost gas sensor nodes for the detection of hazardous substances in the event of a disaster," 2015 IEEE Topical Conference on Wireless Sensors and Sensor Networks (WiSNet), San Diego, CA, 2015, pp. 59-61.

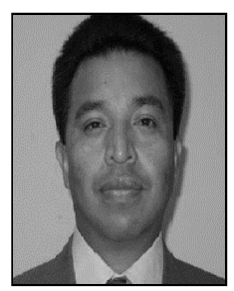

Gregorio Trinidad García, was born in Puebla, Puebla, México. He received the B.S. degree in electronics and the M.S. degree in optoelectronics from the University of Puebla, Puebla. May and December 1998 respectively. He is currently pursuing the Ph.D. degree at the Facultad de Ciencia Físico Matemáticas, BUAP, Puebla, México, where he is working in optoelectronic and embedded system

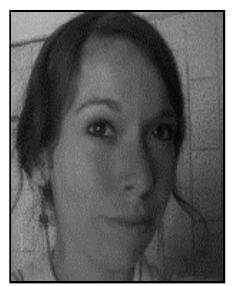

Victoria M. Born in Puebla, Mexico in 1991 Actually study engineering in Computer Science in BUAP. Her interests with science have always gone hand in hand with ecological and social projects and therefore her inclination toward the area of internet of the things, and her studies in the area both software and hardware. In 2016, he has participated in FEPRO with facial recognition systems, for social purposes Currently continues to develop ecological io projects.

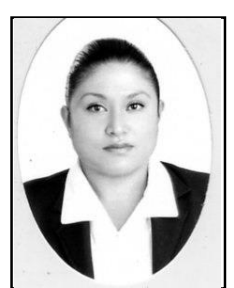

Carmen Nora López Marín, was born in Puebla, Mexico. Her received the B.S. degree in electronics and telecommunication engineer from the University Hispana of Puebla, México in 2004. Her interests with science have always gone hand in hand with ecological and social projects, where her is working in embedded-system

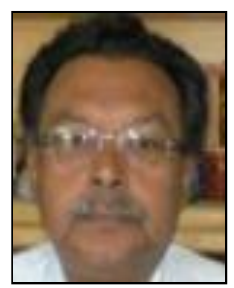

Cortez José Italo, he made studies of Electrical Engineering and Master of Sciences in power station in the Polytechnical Institute of Kiev, Ukraine and Ph.D in Technical Sciences in the North West State University, St. Petersburg, Russian. At the moment it works as titular professor in the Faculty of Computing Science of the Universidad Autónoma de puebla, México.

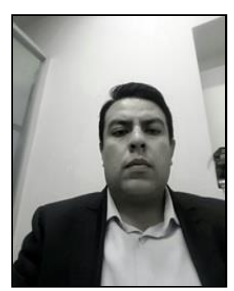

Carlos Armando Ríos Acevedo, was born in Puebla Puebla, Mexico, in 1979. Received the B.S. degree in Computer Science from the University of Puebla, Puebla, and the Master's in Software Engineering from the Graduate School of Puebla in May 2007 respectively. He is certified by Oracle and Microsoft in databases and is a specialist in OLAP cubes and database design and is currently working for the Autonomous University of Puebla.

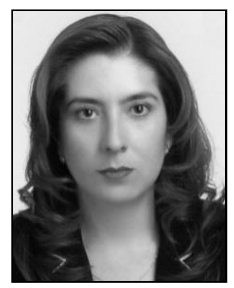

Griselda Saldaña González received a BSc. in Electronics from Universidad Autónoma de Puebla (BUAP), and the MSc. in Electronics Engineering from the Universidad de las Americas Puebla, where she specialized in the area of digital design. She made a Phd. in Computer Science at the National Institute for Astrophysics, Optics and Electronics (INAOE) in Puebla, Mexico, using FPGA devices. She participated as a postdoctoral fellow in the Physics and Mathematics faculty at the BUAP with the group of Particles, Fields and General Relativity. Dr. Saldaña has been researcher in the Mechatronics Division at the Universidad Tecnológica de Puebla since 2001 and collaborates with the Instituto Tecnológico de Puebla since 2014. She is member of PRODEP (México) and has been a member of the 
SNI (2008-2012).

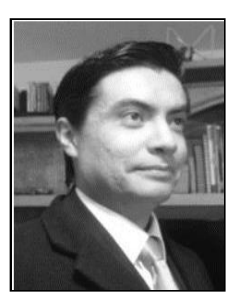

Jose L. Hernandez Ameca. The author was born in Puebla, Pue., Mexico, in 1978. He obtained the degree of Electronic Engineer of the Technological Institute of Puebla in 2001, he received the degree of Master in Computer Science of the Benemérita Autonomous University of Puebla (BUAP) in 2005 and the degree of Doctor in Strategic Planning and Technology Management from the Autonomous Popular University of Puebla in 2017. From 2002 to 2004 he worked as an Engineer in the project implementation department of the IUSACEL digital company. He works as a professor in the faculty of computer sciences of the BUAP since 2007 , where his line of research is automation and robotics.

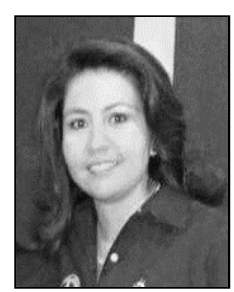

María del Consuelo Molina García, was born was born in Teziutlán, Pue., Mexico, in 1978, her obtained a degree in Computer Science at the Benemérita Universidad Autónoma de Puebla in 2001, he received a Master's degree in Software Engineering from the Universidad Popular del Estado de Puebla (UPAEP) in 2006. He has worked as a professor in the School of Computer Science of the BUAP since 2003, where his research line is Software Engineer. 